\title{
Fabrication of oxygen-defective tungsten oxide nanorods for deep oxidative desulfurization of fuel
}

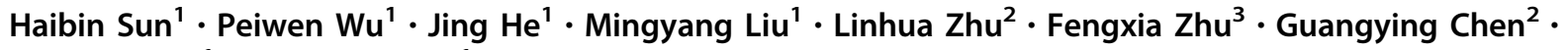 \\ Minqiang $\mathrm{He}^{1} \cdot$ Wenshuai $\mathrm{Zhu}^{1}$
}

Received: 14 April 2018/Published online: 10 October 2018

(C) The Author(s) 2018

\begin{abstract}
Owing to the increasingly serious environmental issues caused by the sulfur burnt in fuel, desulfurization has become an important topic. In this work, an amphiphilic oxygen-defective tungsten oxide was synthesized by a colloidal chemistry method. The amphiphilic property and oxygen defects were well characterized, and the structure of the oxygen-defective tungsten oxide catalyst was investigated. In addition, the catalyst was employed in oxidative desulfurization system of fuel, and deep desulfurization was achieved. It was found that the very high oxidative desulfurization performance of oxygendefective tungsten oxide catalyst resulted from both the amphiphilic property and oxygen defects. This work can provide a strategy for preparation of highly active metal oxide catalysts with oxygen defects in oxidative desulfurization reaction of fuel.
\end{abstract}

Keywords Tungsten oxide $\cdot$ Oxygen vacancies $\cdot$ Oxidative desulfurization $\cdot$ Amphiphilic catalyst

\section{Introduction}

Emission of $\mathrm{SO}_{x}$, originating from the combustion of sulfur compounds in fuel, has become an increasingly serious issue (Wu et al. 2016b; Zhao et al. 2017; Xiao et al. 2016; Lu et al. 2017; Gu et al. 2017; He et al. 2017). Thus, increasingly stringent regulations have been proposed (Xiao et al. 2014; Jiang et al. 2016; Zhu et al. 2015; Zhang et al. 2014a; Xu et al. 2012; Zhu et al. 2013c; Wu et al. 2016a). Generally, the sulfur contents in fuel are limited to $<10 \mathrm{ppm}$ in many countries. To realize such a goal, higher requirements are needed in industrial hydrodesulfurization

Edited by Xiu-Qin Zhu

Handling editor: Wenshuai Zhu

Wenshuai Zhu

zhuws@ujs.edu.cn

1 School of Chemistry and Chemical Engineering, Institute for Energy Research, Jiangsu University, Zhenjiang 212013, China

2 Key Laboratory of Tropical Medicinal Plant Chemistry of Education, Hainan Normal University, Haikou 571158, China

3 School of Chemistry and Chemical Engineering, Huaiyin Normal University, Huaian 223001, China
(HDS), such as higher operating temperature and reaction pressure. However, the conventional HDS process is less efficient for aromatic sulfur compounds, making it a choke point for production of ultra-low sulfur fuel oil. Therefore, numerous substitute technologies have been developed, including extractive desulfurization (EDS) (Gao et al. 2013; Li et al. 2012; Chen et al. 2012; Zhao et al. 2016), adsorptive desulfurization (ADS) (Xiao et al. 2015; Khan et al. 2014; Xiong et al. 2016; Xiao et al. 2013), biodesulfurization (BDS) (Ferreira et al. 2017; Aksoy et al. 2014; Zhang et al. 2017a), oxidative desulfurization (ODS) (Wu et al. 2016a, 2017a, b, 2018; Jiang et al. 2017; Xun et al. 2016), etc. Among all the developed substitutes, ODS is a potential one because of its mild reaction conditions and high activity to aromatic sulfur compounds. The above advantages make ODS a promising complementary method to the HDS process (McNamara et al. 2013; Jiang et al. 2016, 2017; Li et al. 2016; Miao et al. 2016).

To achieve a high ODS efficiency, an important issue is finding a highly active catalyst. Currently, task-specific ionic liquids (TSILs) (Zhu et al. 2013d; Kulkarni and Afonso 2010; Zhang et al. 2013; Wishart 2009; Zhu et al. 2013c; Jiang et al. 2015b), metal oxides (Xiao et al. 2016; He et al. 2015; Gonzalez et al. 2017; Rodriguez-Gattorno et al. 2009), polyoxometalates (Zhang et al. 2017b; Yang 
et al. 2017), titanium silicalites (Kong et al. 2006; Feng et al. 2017; Du et al. 2017; Shen et al. 2015), and so on have been widely employed in ODS. Among all the reported catalysts, a metal oxide is an eye-catching one, which is especially true when it comes to tungsten oxide. However, based on a previous report, stoichiometric tungsten oxide rarely shows excellent catalytic performance (He et al. 2017). For non-stoichiometric tungsten oxide, because of the variable valences of $\mathrm{W}$, the tungsten oxide can be readily reacted with an oxidant to form a reactive intermediate for the further oxidation process. Nevertheless, for tungsten oxide catalysts, bulk tungsten oxide rarely has satisfying catalytic performance because of the poor exposure of catalytically active sites. Thus, numerous strategies have been adopted to improve this situation. For example, our group employed graphene-like hexagonal boron nitride as a support for dispersion of tungsten oxide to prepare the $\mathrm{WO}_{x}$ in nanoparticle form (Wu et al. 2015b). However, such strategy is less universally applicable and involves high-temperature treatment. On the other hand, the poor catalytic activity of the bulk tungsten oxide in ODS has mainly originated from the strong hydrophilic property of tungsten oxide, making the catalyst difficult to contact with the fuel oil.

Recently, preparation of metal/metal oxides by colloidal preparation methods has attracted increasing attention because of the successful synthesis of nano-sized materials and a controllable process (Guo et al. 2013; Jiang et al. 2015a; Wu et al. 2015a; Zhang et al. 2014b; Zhu et al. 2013a, b). Moreover, such a process is usually realized in an oil phase, making organic ligands abundant on the surfaces of metal/metal oxide. This property allows the catalyst to be easily dispersed in fuel oil. Thus, when a colloidal chemistry method in an oil phase is employed for the preparation of tungsten oxide materials, there will be not only a catalyst with nanosize, but also it will drive the catalyst to be easily dispersed in fuel oil, which gives rise to an excellent desulfurization performance.

In this work, a method for colloidal preparation of nonstoichiometric tungsten oxide $\left(\mathrm{W}_{18} \mathrm{O}_{49}\right)$ nanorods was employed. The obtained catalyst was characterized as having abundant organic ligands on its surface. Because of the existence of organic ligands on the surface, the obtained $\mathrm{W}_{18} \mathrm{O}_{49}$ nanorods show excellent lipophilicity, making the catalyst easy to contact with fuel oil and lead to a high desulfurization performance. Additionally, effects of oxidant amount and reaction temperature were investigated in detail. The current work may provide a strategy for preparation of highly active and amphipathic catalyst for deep oxidative desulfurization.

\section{Experimental}

\subsection{Material}

Oleylamine (OAM, 90\%), oleic acid (OAC, AR), tungsten hexachloride $\left(\mathrm{WCl}_{6}, \mathrm{AR}\right)$, dibenzothiophene (DBT, 99\%), and tetradecane (AR) were purchased from Sigma-Aldrich and used without further purification. Ethanol $\left(\mathrm{C}_{2} \mathrm{H}_{5} \mathrm{OH}\right.$, AR), $n$-hexane (AR), and hydrogen peroxide $\left(\mathrm{H}_{2} \mathrm{O}_{2}, 30\right.$ wt $\%$ ) were obtained from Sinopharm Chemical Reagent Co., Ltd. China.

\subsection{Preparation of catalyst}

\subsubsection{Preparation of $\mathrm{W}_{18} \mathrm{O}_{49}-\mathrm{MC}$}

OAM- and OAC-modified $\mathrm{W}_{18} \mathrm{O}_{49}$ was prepared as follows: typically, $0.2 \mathrm{~g}$ of $\mathrm{WCl}_{6}$ was dissolved in $10 \mathrm{~mL}$ of ethanol and stirred for 10 min under $\mathrm{N}_{2}$. Afterward, a mixture of $0.5 \mathrm{~mL}$ of OAM and $0.5 \mathrm{~mL}$ of OAC was added to the solution drop by drop and stirred for another $10 \mathrm{~min}$. Then, the mixed solution was transferred to a $20-\mathrm{mL}$ autoclave. Subsequently, the autoclave was placed in an oven at $180{ }^{\circ} \mathrm{C}$ for $24 \mathrm{~h}$ followed by natural cooling to room temperature. The obtained product was separated by centrifugation at a speed of 10,000 rpm for $10 \mathrm{~min}$. Then, the product was washed with ethanol and re-dispersed in cyclohexane for three times. Finally, the product was placed in a vacuum oven at $50{ }^{\circ} \mathrm{C}$ for $10 \mathrm{~h}$ to obtain the product. The obtained black-blue catalyst was denoted as $\mathrm{W}_{18} \mathrm{O}_{49}$-MC.

\subsubsection{Preparation of $\mathrm{W}_{18} \mathrm{O}_{49}-\mathrm{E}$}

The preparation process for $\mathrm{W}_{18} \mathrm{O}_{49}$-E is similar to that of $\mathrm{W}_{18} \mathrm{O}_{49}-\mathrm{MC}$, except without adding OAM and OAC during the preparation process.

\subsection{Characterization}

A Nicolet Nexus 470 Fourier transform infrared spectrometer was used to collect the Fourier transform infrared spectra (FT-IR). They were recorded at room temperature with $\mathrm{KBr}$ pellets. X-ray diffraction (XRD) patterns were from a $\mathrm{D} 8 \mathrm{Advance}$ with $\mathrm{Cu} \mathrm{K} \alpha$ radiation and ranged from $10^{\circ}$ to $80^{\circ}$ with a scanning rate of $7 \% \mathrm{~min}$. A Hitachi H-700 was used for transmission electron microscopy (TEM). Scanning electron microscopy (SEM) images were recorded with a JSM-7001F thermal field emission scanning electron microscope at $2-15 \mathrm{keV}$ accelerating voltage. Ultraviolet-visible diffuse reflectance spectra (UV-vis DRS) were recorded by a Shimadzu UV-2450 
spectrophotometer with a spherical diffuse reflectance accessory. The scanning range was $200-800 \mathrm{~nm}$ and $\mathrm{BaSO}_{4}$ was employed as background. The XPS results were collected on a PHI 3056 spectrometer with an Al anode source.

\subsection{Oxidative desulfurization experiments}

\subsubsection{Preparation of model oil}

The DBT-containing model oil was prepared by the following process: DBT was dissolved in $n$-octane to make the sulfur concentration of $500 \mathrm{ppm}$. Afterward, additional tetradecane was added to a concentration of $4000 \mathrm{ppm}$ as the internal standard.

\subsubsection{Catalysis experiments}

The catalytic oxidative desulfurization was carried out as follows: firstly, a certain amount of catalyst was added to a 40-mL homemade two-neck flask. Afterward, $5 \mathrm{~mL}$ of DBT-containing model oil was added, followed by a certain amount of $\mathrm{H}_{2} \mathrm{O}_{2}$. Then, the flask was placed in an oil bath at the required reaction temperature and magnetically stirred. After required reaction times, the oil was allowed to stand for 1 min to allow the separation of the oil and catalyst phases, and $1 \mu \mathrm{L}$ of the upper oil phase was taken for further analysis.

\subsubsection{Analysis of sulfur content}

The sulfur content in fuel oil was analyzed with an Agilent 7890 gas chromatograph equipped with an HP-5 column. The HP-5 column is $30 \mathrm{~m}$ long $\times 0.32 \mathrm{~mm}$ inner diameter, $0.25 \mu \mathrm{m}$ of film thickness. The sulfur removal was calculated by Eq. (1).

Sulfur removal $(\%)=\left(1-C_{\mathrm{A}} / C_{0}\right) \times 100 \%$

where $C_{\mathrm{A}}$ stands for the sulfur concentration of model oil and $C_{0}$ is the initial sulfur concentration.

\section{Results and discussion}

\subsection{Raman characterization}

To determine the structure of the $\mathrm{W}_{18} \mathrm{O}_{49}$-MC catalyst, the Raman spectrum was obtained and is shown in Fig. 1. The results show that the peaks around $810 \mathrm{~cm}^{-1}$ and $700 \mathrm{~cm}^{-1}$ are the characteristic peaks of $\mathrm{O}-\mathrm{W}-\mathrm{O}$, and the very small peak at $950 \mathrm{~cm}^{-1}$ is due to the stretching modes of $\mathrm{O}-\mathrm{W}-\mathrm{O}$. A strong peak around $230 \mathrm{~cm}^{-1}$ is from the bending vibration mode of $\mathrm{O}-\mathrm{W}-\mathrm{O}$ (He et al. 2017). The

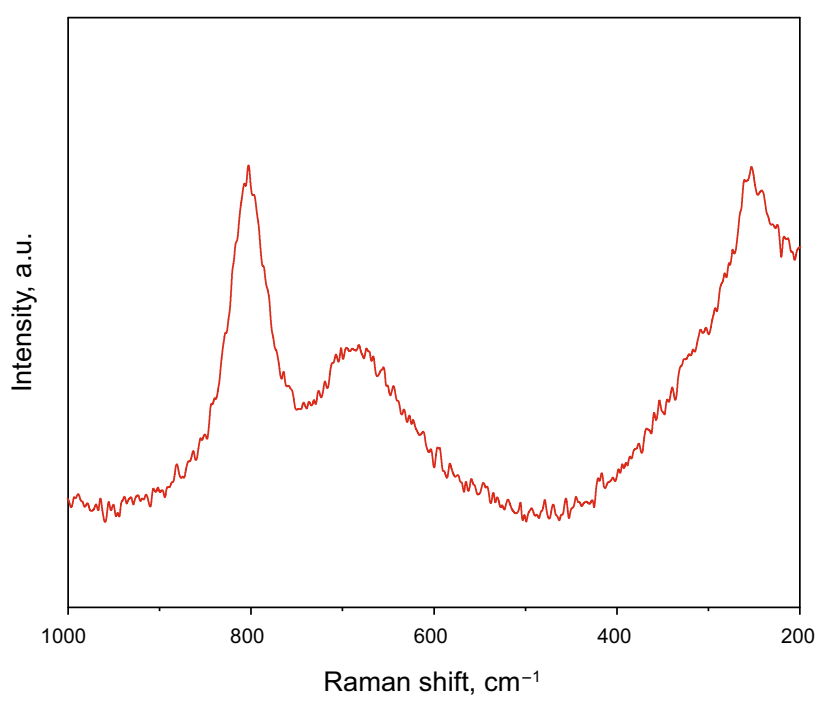

Fig. 1 Raman spectrum of $\mathrm{W}_{18} \mathrm{O}_{49}-\mathrm{MC}$

Raman spectrum is a solid evidence of achievement of the $\mathrm{W}_{18} \mathrm{O}_{49}$ structure by the current method.

\section{2 $\mathrm{N}_{2}$ adsorption-desorption isotherm analysis and SEM}

The physical structure of the $\mathrm{W}_{18} \mathrm{O}_{49}-\mathrm{MC}$ was characterized by its $\mathrm{N}_{2}$ adsorption-desorption isotherm at $-196{ }^{\circ} \mathrm{C}$. The specific surface area of the sample is low, only $3.8 \mathrm{~m}^{2} /$ $\mathrm{g}$, indicating that the current preparation method does not give rise to a high specific surface area due to the existence of organic ligands on the surface. The corresponding SEM image (Fig. 2) verified the conclusion that the nanorods were seriously agglomerated. The result indicates that the prepared $\mathrm{W}_{18} \mathrm{O}_{49}-\mathrm{MC}$ has an organic ligand-covered structure.

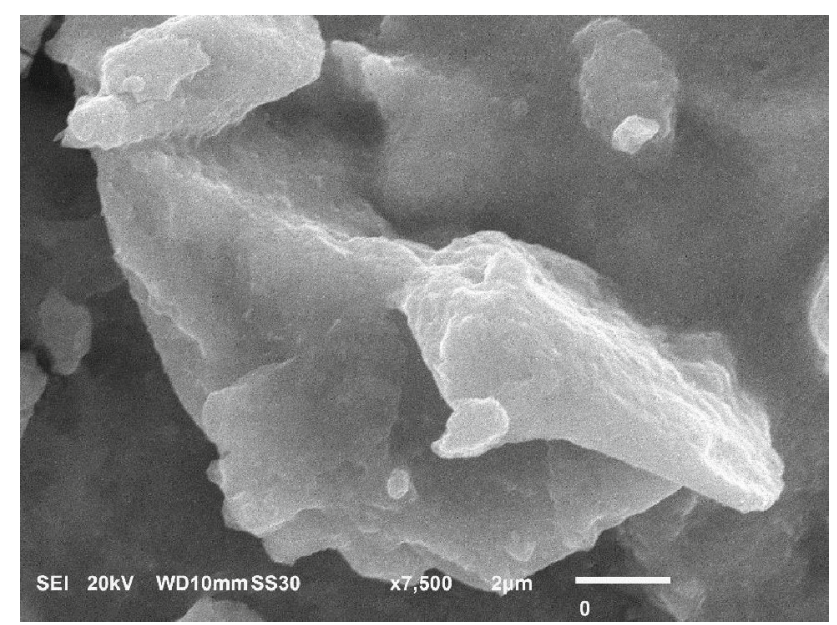

Fig. 2 SEM image of $\mathrm{W}_{18} \mathrm{O}_{49}-\mathrm{MC}$ 


\subsection{XRD characterization of $\mathrm{W}_{18} \mathrm{O}_{49}-\mathrm{MC}$ catalyst}

More information about the catalyst is gained from its XRD pattern in Fig. 3. After a careful comparison with the standard powder diffraction file card (PDF\#84-1516), the prepared $\mathrm{W}_{18} \mathrm{O}_{49}$-MC was found to be a monoclinic phase. It is noteworthy that the intensities of the peaks are relatively weak, and all peaks are wide. The result illustrates that the prepared catalyst has an ultra-small particle size, which is beneficial to the exposure of the catalytically active sites.

\subsection{UV-vis DRS characterization of $\mathrm{W}_{18} \mathrm{O}_{49}$-MC catalyst}

To verify the oxygen-defective structure of the $\mathrm{W}_{18} \mathrm{O}_{49^{-}}$ MC catalyst, the UV-visible diffuse reflectance spectrum was obtained and is shown in Fig. 4. In addition to the characteristic peak around $230 \mathrm{~nm}$, a widened adsorption in the visible light range is clearly detected. The result demonstrates that the prepared tungsten oxide has a nonstoichiometric structure and contains abundant oxygen defects, which is inline with the XRD characterization result.

\subsection{FT-IR characterization of the catalyst}

To analyze the structure of the prepared catalysts, especially the surface organic ligands, we tested the FT-IR spectra of the both prepared catalysts (Fig. 5). As can be seen from the spectra, both samples showed characteristic peaks around 950,853 , and $755-600 \mathrm{~cm}^{-1}$, respectively, being assigned to $\mathrm{W}=\mathrm{O}, \mathrm{W}-\mathrm{O}_{\mathrm{b}}-\mathrm{W}$, and $\mathrm{O}-\mathrm{W}-\mathrm{O}$ bonds, verifying the structure of tungsten oxide. The very obvious differences between the samples are the adsorption peaks

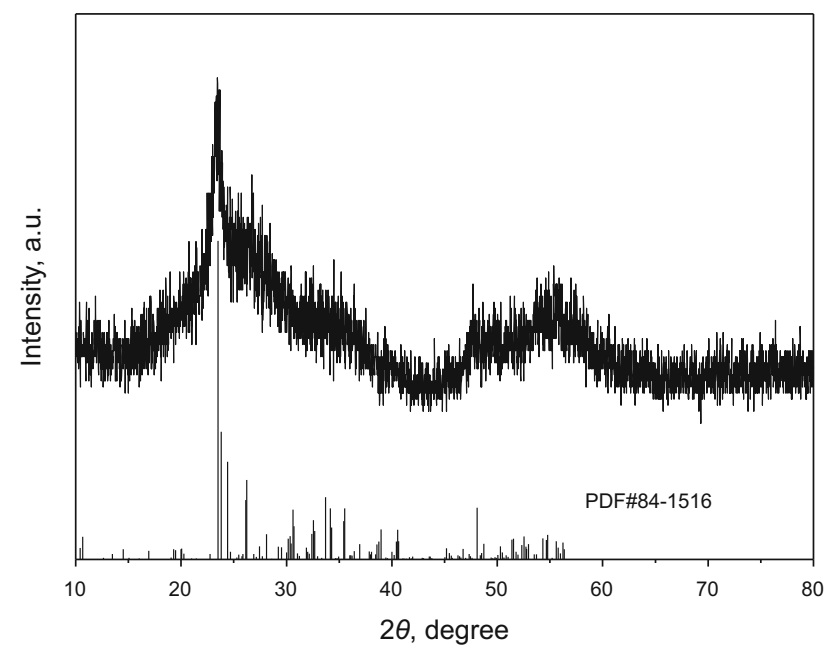

Fig. $3 \mathrm{XRD}$ characterization of the $\mathrm{W}_{18} \mathrm{O}_{49}-\mathrm{MC}$ catalyst

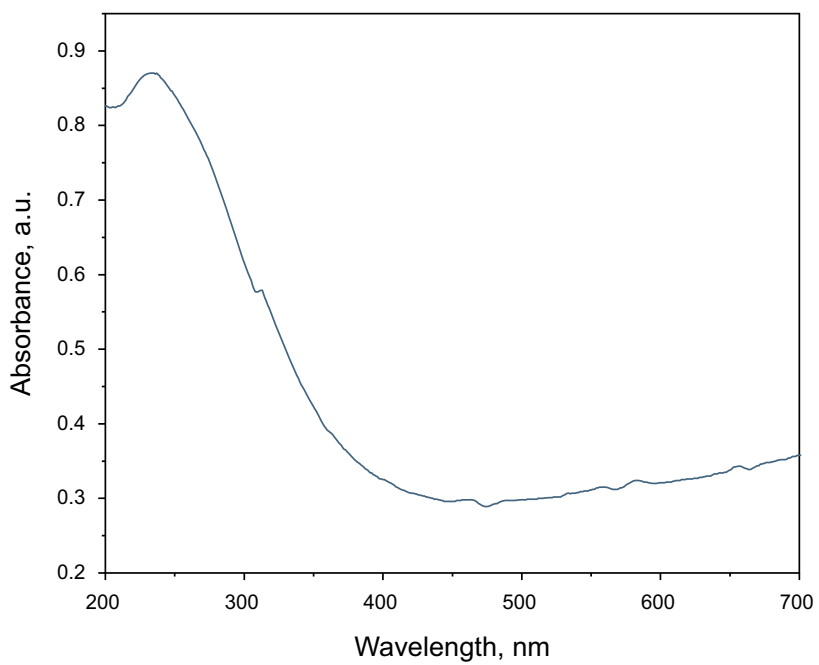

Fig. 4 UV-vis DRS spectrum of $\mathrm{W}_{18} \mathrm{O}_{49}$-MC catalyst

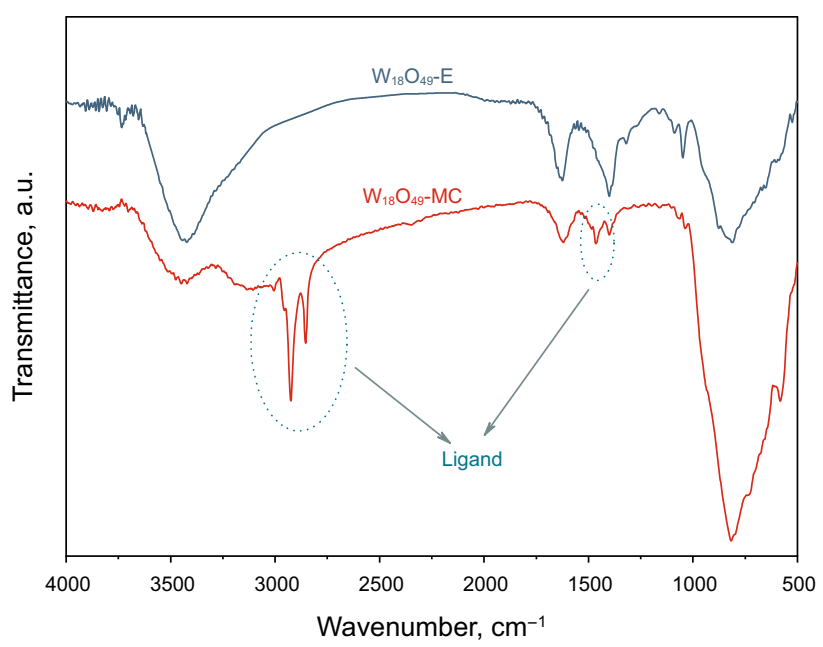

Fig. 5 FT-IR spectra of prepared samples

around $1450-1650 \mathrm{~cm}^{-1}$ and $2750-2900 \mathrm{~cm}^{-1}$. As we can see from the Fig. 5, compared with $\mathrm{W}_{18} \mathrm{O}_{49}-\mathrm{E}$, the $\mathrm{W}_{18} \mathrm{O}_{49^{-}}$ MC sample showed a group of strong peaks around $2750-2900 \mathrm{~cm}^{-1}$, attributing to $-\mathrm{CH}_{2}-$ and $-\mathrm{CH}_{3}$ groups, while the peak $1465 \mathrm{~cm}^{-1}$ is assigned to the bending vibration mode of $-\mathrm{CH}_{2}-$. Additionally, the peak around $1630 \mathrm{~cm}^{-1}$ in $\mathrm{W}_{18} \mathrm{O}_{49}-\mathrm{MC}$ is ascribed to $-\mathrm{NH}_{2}$ groups of the organic ligands. The characterization verifies that the surface of $\mathrm{W}_{18} \mathrm{O}_{49}$-MC is covered by organic ligands, while the surface of $\mathrm{W}_{18} \mathrm{O}_{49}-\mathrm{E}$ is covered by $-\mathrm{OH}$ groups (the strong peak around $3400 \mathrm{~cm}^{-1}$ ). As known, in oxidative desulfurization with $\mathrm{H}_{2} \mathrm{O}_{2}$ as the oxidant, the contact of catalyst, oil, and $\mathrm{H}_{2} \mathrm{O}_{2}$ is essential to a high desulfurization performance. From the structures of the catalysts, we can draw a conclusion that the $\mathrm{W}_{18} \mathrm{O}_{49}$ - $\mathrm{E}$ is a hydrophilic catalyst and $\mathrm{W}_{18} \mathrm{O}_{49}-\mathrm{MC}$ is an amphipathic one. 

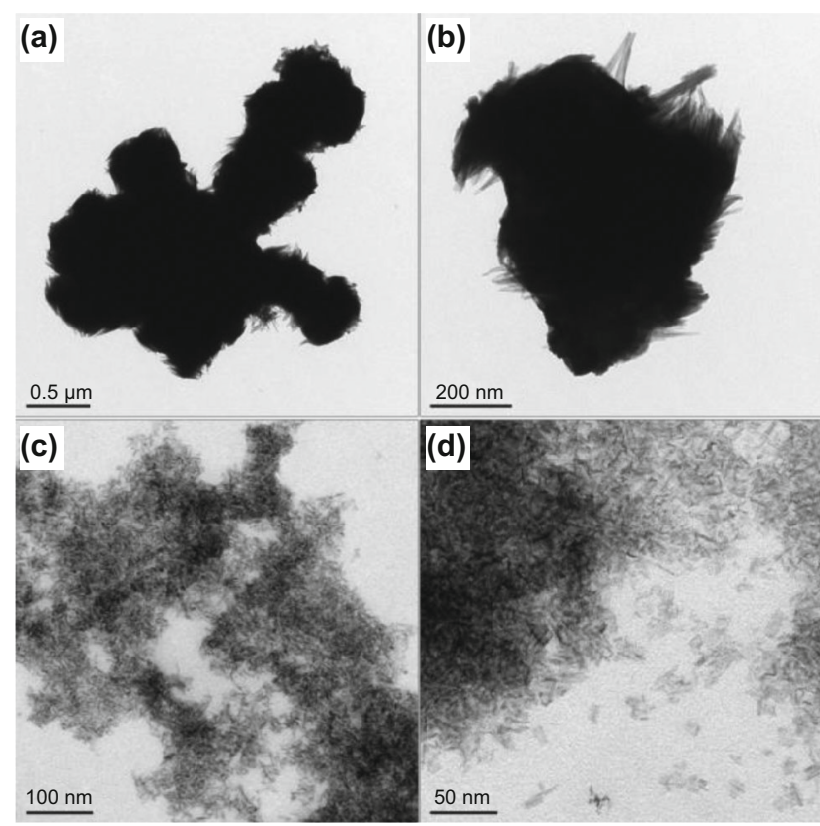

Fig. 6 TEM characterizations of the catalyst. a, b TEM images of $\mathrm{W}_{18} \mathrm{O}_{49}-\mathrm{E}$; $\mathbf{c}, \mathbf{d}$ TEM images of $\mathrm{W}_{18} \mathrm{O}_{49}$-MC

\subsection{TEM image of the $\mathrm{W}_{18} \mathrm{O}_{49}-\mathrm{MC}$ catalyst}

To further confirm the conclusion, TEM characterization was performed, and the results are shown in Fig. 6. The results showed that the $\mathrm{W}_{18} \mathrm{O}_{49}$-E catalyst held an undispersed status with a size $>500 \mathrm{~nm}$. From the TEM image of $\mathrm{W}_{18} \mathrm{O}_{49}-\mathrm{MC}$, the tungsten oxide was well dispersed and it is in the form of nanorods. It is known that in liquid-solid heterogeneous catalytic system, contact between catalyst and substrates is vital to the catalytic performance because of the exposure of catalytically active sites. The ligandabundant $\mathrm{W}_{18} \mathrm{O}_{49}-\mathrm{MC}$ catalyst was well dispersed and in the form of nanorods exposing numerous catalytically active sites for oxidative desulfurization.

\subsection{Catalytic oxidative desulfurization performance}

The prepared catalyst was employed in oxidative desulfurization and used $\mathrm{H}_{2} \mathrm{O}_{2}$ as an oxidant. As seen from Fig. 7 compared with ligand-free $\mathrm{W}_{18} \mathrm{O}_{49}-\mathrm{E}, \mathrm{W}_{18} \mathrm{O}_{49}$-MC shows a remarkable boosted oxidative desulfurization that the sulfur removal is enhanced from $52 \%$ to $>98 \%$. Additionally, the dispersion of both catalysts in fuel oil is shown in the inset of Fig. 7. The $\mathrm{W}_{18} \mathrm{O}_{49}$-MC is well dispersed in the model oil, while the $\mathrm{W}_{18} \mathrm{O}_{49}$-E catalyst aggregated in the model oil. The phenomenon is in accordance with the TEM characterization that $\mathrm{W}_{18} \mathrm{O}_{49}$-MC is well contacted with substrates for an enhanced oxidative desulfurization performance.

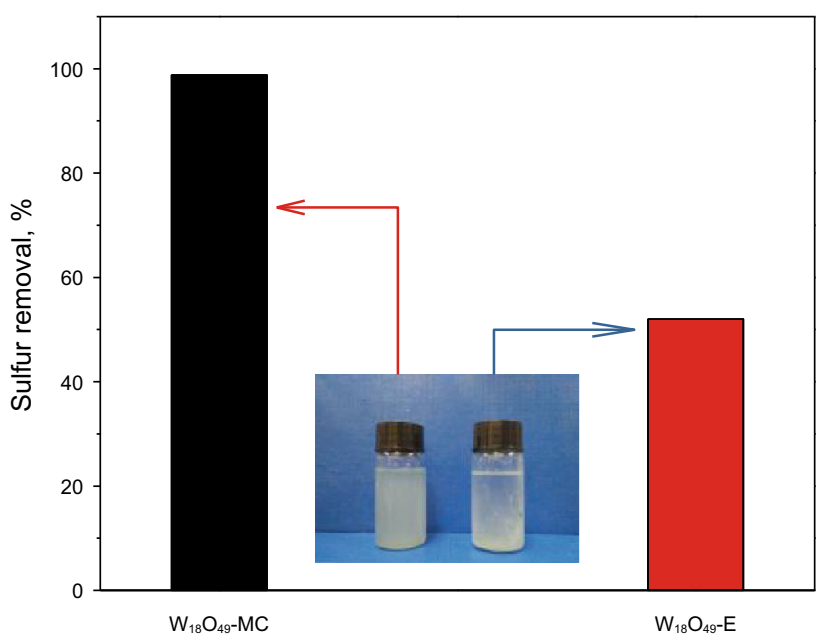

Fig. 7 Catalytic oxidative desulfurization performance of the catalysts. The inset is the dispersion of the catalysts in model oil. Reaction conditions: $\quad m($ Catal. $)=0.01 \mathrm{~g} ; \quad V($ oil $)=5 \mathrm{~mL} ; \quad T=60{ }^{\circ} \mathrm{C}$; $t=40 \mathrm{~min} ;$ molar ratio $n(\mathrm{O}) / n(\mathrm{~S})=3$

\subsection{Effect of $n(0) / n(S)$ on sulfur removal}

The effect of the amount of $\mathrm{H}_{2} \mathrm{O}_{2}$ used was studied to optimize the reaction conditions, and the results are shown in Fig. 8. From the result, it can be seen that when molar ratio of $\mathrm{H}_{2} \mathrm{O}_{2}$ to sulfur (denoted as $n(\mathrm{O}) / n(\mathrm{~S})$ ) was 2, which is the stoichiometric requirement for oxidation of DBT to $\mathrm{DBTO}_{2}$, the sulfur removal was $90 \%$ at a reaction time of $60 \mathrm{~min}$ and deep desulfurization was not achieved. This may be attributed to the self-decomposition of $\mathrm{H}_{2} \mathrm{O}_{2}$ under high reaction temperatures. When the $n(\mathrm{O}) / n(\mathrm{~S})$ increased from 2 to 3, a deep desulfurization performance will gain after only $40 \mathrm{~min}$ of reaction time. A further increase in the amount of $\mathrm{H}_{2} \mathrm{O}_{2}$ did not result in a significant increment in desulfurization performance. As such, we choose $n(\mathrm{O}) /$

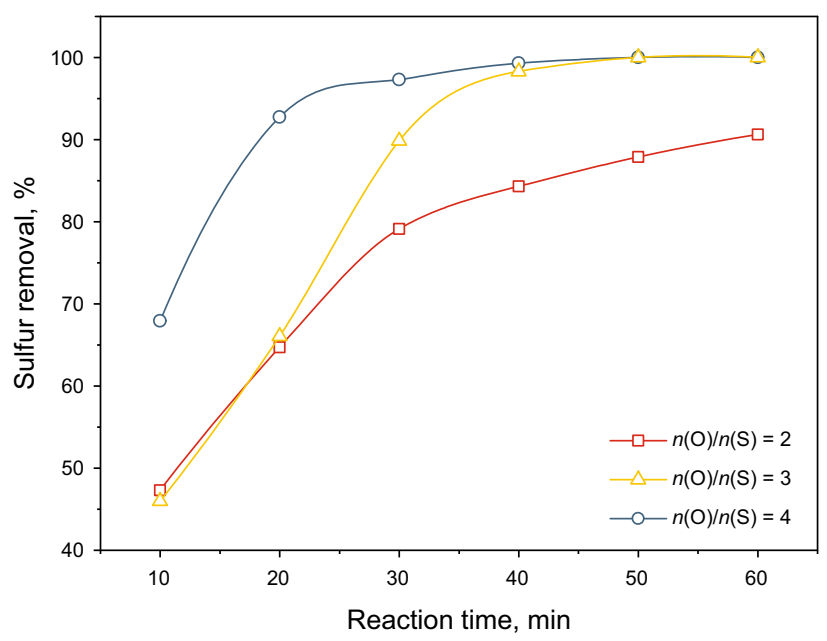

Fig. 8 Effect of $n(\mathrm{O}) / n(\mathrm{~S})$ on sulfur removal. Reaction conditions: $m$ (Catal. $)=0.01 \mathrm{~g} ; V($ oil $)=5 \mathrm{~mL} ; T=60^{\circ} \mathrm{C}$ 
$n(\mathrm{~S})$ was 3 as the optimized $\mathrm{H}_{2} \mathrm{O}_{2}$ amount and 40 min as the proper reaction time.

\subsection{Effect of reaction temperature on sulfur removal}

The influence of reaction temperature on sulfur removal is shown in Fig. 9. It can be seen that the sulfur removal increased with an increase in reaction temperature. Although when the reaction temperature was $50{ }^{\circ} \mathrm{C}$, a $\sim 100 \%$ of sulfur removal was obtained after a reaction of $60 \mathrm{~min}$, the longer reaction time is less favored. Hence, we chose $60{ }^{\circ} \mathrm{C}$ as the most optimized reaction temperature.

\subsection{Recycling performance of the catalyst}

In catalysis, recycling performance is an important parameter for evaluating a catalyst. Thus, the recycling performance of the $\mathrm{W}_{16} \mathrm{O}_{49}$-MC catalyst was investigated and is shown in Fig. 10. Typically, the recycling process of the catalyst was realized by the following steps: after each run, the catalyst phase was separated by pouring out the oil phase after centrifugation. Then, the catalyst was dried in an oven at $120{ }^{\circ} \mathrm{C}$ overnight. Afterward, fresh oil and oxidant were added for the next run. The catalyst can be cycled five times without significant decrease in the catalytic activity. The result illustrates that the catalyst is a promisingly stable one.

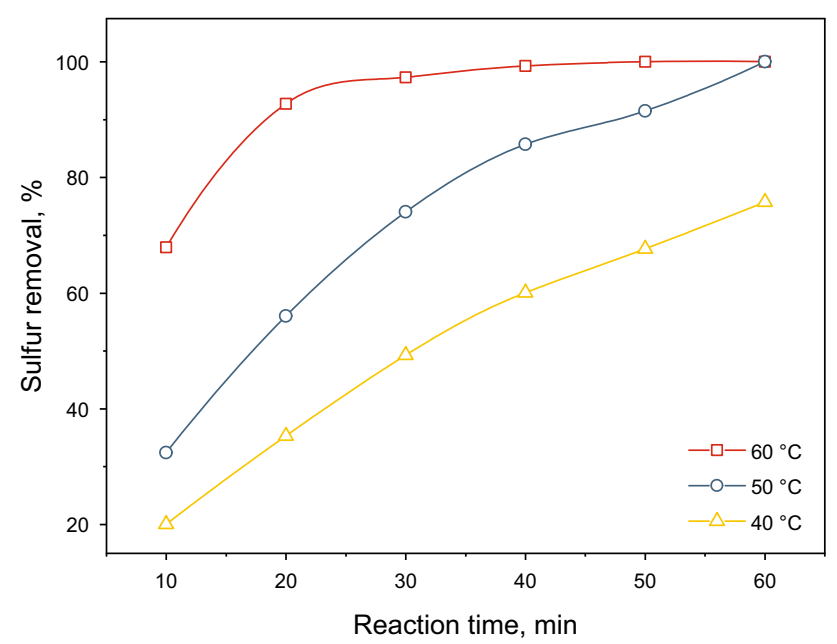

Fig. 9 Effect of reaction temperature on sulfur removal. Reaction conditions: $m($ Catal. $)=0.01 \mathrm{~g} ; V($ oil $)=5 \mathrm{~mL} ; n(\mathrm{O}) / n(\mathrm{~S})=3$

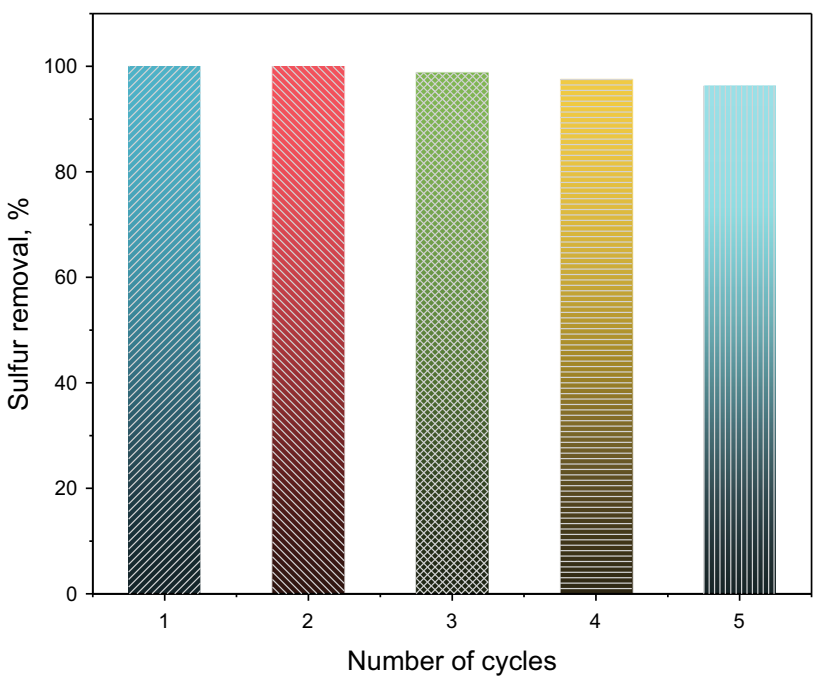

Fig. 10 Recycling performance of the catalyst. Reaction conditions: $m($ Catal. $)=0.01 \mathrm{~g} ; T=60^{\circ} \mathrm{C} ; V($ oil $)=5 \mathrm{~mL} ; n(\mathrm{O}) / n(\mathrm{~S})=3$

\section{Conclusion}

A ligand-covered tungsten oxide with oxygen defects was successfully obtained by a colloidal chemistry method. The amphipathic property and oxygen defects were well characterized, and the structure of the catalyst was determined by a series of characterization methods. It was found that with the presence of oleylamine and oleic acid, the obtained tungsten oxide was in the form of nanorods, which is beneficial to the contact between catalyst and substrates. Furthermore, the obtained catalyst was employed as an efficient catalyst in oxidative desulfurization. With optimized reaction conditions, a deep desulfurization performance was obtained, making the $\mathrm{S}$ content lower than $10 \mathrm{ppm}$. The very excellent oxidative desulfurization performance mainly originates from the amphipathic property and oxygen defects of the catalyst. This work may provide a strategy for the preparation of highly active oxidative desulfurization metal oxide catalysts.

Acknowledgements The authors are grateful for financial support by Students' Platform for innovation and entrepreneurship training of Jiangsu Province (201810299008z), the National Nature Science Foundation of China (Nos. 21576122, 21722604, 21766007), Key Laboratory of Tropical Medicinal Plant Chemistry of Ministry of Education, Hainan Normal University (20150376), and the Natural Science Foundation of Jiangsu Provincial Department of Education (17KJA150002).

Open Access This article is distributed under the terms of the Creative Commons Attribution 4.0 International License (http://creative commons.org/licenses/by/4.0/), which permits unrestricted use, distribution, and reproduction in any medium, provided you give appropriate credit to the original author(s) and the source, provide a link to the Creative Commons license, and indicate if changes were made. 


\section{References}

Aksoy DO, Aytar P, Toptas Y, Cabuk A, Koca S, Koca H. Physical and physicochemical cleaning of lignite and the effect of cleaning on biodesulfurization. Fuel. 2014;132:158-64. https:// doi.org/10.1016/j.fuel.2014.04.090.

Chen X, Liu G, Yuan S, Asumana C, Wang W, Yu G. Extractive desulfurization of fuel oils with thiazolium-based ionic liquids. Separ Sci Technol. 2012;47(6):819-26. https://doi.org/10.1080/ 01496395.2011 .637281$.

Du Q, Guo Y, Duan H, Li H, Chen Y, Liu H. Synthesis of hierarchical TS-1 zeolite via a novel three-step crystallization method and its excellent catalytic performance in oxidative desulfurization. Fuel. 2017;188:232-8. https://doi.org/10.1016/j.fuel.2016.10. 045.

Feng X, Sheng N, Liu Y, Chen X, Chen D, Yang C, et al. Simultaneously enhanced stability and selectivity for propene epoxidation with $\mathrm{H}_{2}$ and $\mathrm{O}_{2}$ on Au catalysts supported on nanocrystalline mesoporous TS-1. ACS Catal. 2017;7(4):2668-75. https://doi.org/10.1021/acscatal.6b03498.

Ferreira P, Sousa SF, Fernandes PA, Ramos MJ. Improving the catalytic power of the DszD enzyme for the biodesulfurization of crude oil and derivatives. Chem-Eur J. 2017;23(68):17231-41. https://doi.org/10.1002/chem.201704057.

Gao J, Meng H, Lu Y, Zhang H, Li C. A carbonium pseudo ionic liquid with excellent extractive desulfurization performance. AIChE J. 2013;59(3):948-58. https://doi.org/10.1002/aic.13869.

Gonzalez J, Wang JA, Chen LF, Manriquez ME, Dominguez JM. Structural defects, Lewis acidity, and catalysis properties of mesostructured $\mathrm{WO}_{3} / \mathrm{SBA}-15$ nanocatalysts. J Phys Chem C. 2017;121(43):23988-99. https://doi.org/10.1021/acs.jpcc. $7 \mathrm{~b} 06373$.

Gu Q, Wen G, Ding Y, Wu K-H, Chen C, Su D. Reduced graphene oxide: a metal-free catalyst for aerobic oxidative desulfurization. Green Chem. 2017;19(4):1175-81. https://doi.org/10.1039/ c6gc02894b.

Guo S, Li D, Zhu H, Zhang S, Markovic NM, Stamenkovic VR, et al. $\mathrm{FePt}$ and CoPt nanowires as efficient catalysts for the oxygen reduction reaction. Angew Chem Int Edit. 2013;52(12):3465-8. https://doi.org/10.1002/anie.201209871.

He J, Liu H, Xu B, Wang X. Highly flexible sub-1 nm tungsten oxide nanobelts as efficient desulfurization catalysts. Small. 2015;11(9-10):1144-9. https://doi.org/10.1002/smll.201401273.

He J, Wu P, Wu Y, Li H, Jiang W, Xun S, et al. Taming interfacial oxygen vacancies of amphiphilic tungsten oxide for enhanced catalysis in oxidative desulfurization. ACS Sustain Chem Eng. 2017;5(10):8930-8. https://doi.org/10.1021/acssuschemeng. $7 \mathrm{~b} 01741$.

Jiang G, Zhu H, Zhang X, Shen B, Wu L, Zhang S, et al. Core/shell face-centered tetragonal $\mathrm{FePd} / \mathrm{Pd}$ nanoparticles as an efficient non-Pt catalyst for the oxygen reduction reaction. ACS Nano. 2015a;9(11):11014-22. https://doi.org/10.1021/acsnano. $5 \mathrm{~b} 04361$.

Jiang W, Zhu W, Li H, Wang X, Yin S, Chang Y, et al. Temperatureresponsive ionic liquid extraction and separation of the aromatic sulfur compounds. Fuel. 2015b;140:590-6. https://doi.org/10. 1016/j.fuel.2014.09.083.

Jiang B, Yang H, Zhang L, Zhang R, Sun Y, Huang Y. Efficient oxidative desulfurization of diesel fuel using amide-based ionic liquids. Chem Eng J. 2016;283:89-96. https://doi.org/10.1016/j. cej.2015.07.070.

Jiang W, Zheng D, Xun S, Qin Y, Lu Q, Zhu W, et al. Polyoxometalate-based ionic liquid supported on graphite carbon induced solvent-free ultra-deep oxidative desulfurization of model fuels. Fuel. 2017;190:1-9. https://doi.org/10.1016/j.fuel.2016.11.024.
Khan NA, Hasan Z, Jhung SH. Ionic liquids supported on metalorganic frameworks: remarkable adsorbents for adsorptive desulfurization. Chem-Eur J. 2014;20(2):376-80. https://doi. org/10.1002/chem.201304291.

Kong LY, Li G, Wang XS, Wu B. Oxidative desulfurization of organic sulfur in gasoline over Ag/TS-1. Energy Fuel. 2006;20(3):896-902. https://doi.org/10.1021/ef050252r.

Kulkarni PS, Afonso CAM. Deep desulfurization of diesel fuel using ionic liquids: current status and future challenges. Green Chem. 2010;12(7):1139-49. https://doi.org/10.1039/c002113j.

Li F-T, Kou C-G, Sun Z-M, Hao Y-J, Liu R-H, Zhao D-S. Deep extractive and oxidative desulfurization of dibenzothiophene with $\mathrm{C}_{5} \mathrm{H}_{9} \mathrm{NO} \cdot \mathrm{SnCl}_{2}$ coordinated ionic liquid. J Hazard Mater. 2012;205:164-70. https://doi.org/10.1016/j.jhazmat.2011.12. 054.

Li S-W, Gao R-M, Zhang R-L, Zhao J-S. Template method for a hybrid catalyst material POM@MOF-199 anchored on MCM41: highly oxidative desulfurization of DBT under molecular oxygen. Fuel. 2016;184:18-27. https://doi.org/10.1016/j.fuel. 2016.06.132.

Lu S-X, Zhong H, Mo D-M, Hu Z, Zhou H-L, Yao Y. A H-titanate nanotube with superior oxidative desulfurization selectivity. Green Chem. 2017;19(5):1371-7. https://doi.org/10.1039/ c6gc03573f.

McNamara ND, Neumann GT, Masko ET, Urban JA, Hicks JC. Catalytic performance and stability of (V) MIL-47 and (Ti) MIL125 in the oxidative desulfurization of heterocyclic aromatic sulfur compounds. J Catal. 2013;305:217-26. https://doi.org/10. 1016/j.jcat.2013.05.021.

Miao G, Huang D, Ren X, Li X, Li Z, Xiao J. Visible-light induced photocatalytic oxidative desulfurization using $\mathrm{BiVO}_{4} / \mathrm{C}_{3} \mathrm{~N}_{4} @$ $\mathrm{SiO}_{2}$ with air/cumene hydroperoxide under ambient conditions. Appl Catal B-Environ. 2016;192:72-9. https://doi.org/10.1016/j. apcatb.2016.03.033

Rodriguez-Gattorno G, Galano A, Torres-Garcia E. Surface acidbasic properties of $\mathrm{WO}_{x}-\mathrm{ZrO}_{2}$ and catalytic efficiency in oxidative desulfurization. Appl Catal B-Environ. 2009;92(1-2):1-8. https://doi.org/10.1016/j.apcatb.2009.07.031.

Shen C, Wang YJ, Xu JH, Luo GS. Synthesis of TS- 1 on porous glass beads for catalytic oxidative desulfurization. Chem Eng J. 2015;259:552-61. https://doi.org/10.1016/j.cej.2014.08.027.

Wishart JF. Energy applications of ionic liquids. Energy Environ Sci. 2009;2(9):956-61. https://doi.org/10.1039/b906273d.

Wu L, Li Q, Wu CH, Zhu H, Mendoza-Garcia A, Shen B, et al. Stable cobalt nanoparticles and their monolayer array as an efficient electrocatalyst for oxygen evolution reaction. J Am Chem Soc. 2015a;137(22):7071-4. https://doi.org/10.1021/jacs. $5 \mathrm{~b} 04142$.

Wu P, Zhu W, Wei A, Dai B, Chao Y, Li C, et al. Controllable fabrication of tungsten oxide nanoparticles confined in grapheneanalogous boron nitride as an efficient desulfurization catalyst. Chem-Eur J. 2015b;21(43):15421-7. https://doi.org/10.1002/ chem.201501413.

Wu P, Zhu W, Chao Y, Zhang J, Zhang P, Zhu H, et al. A templatefree solvent-mediated synthesis of high surface area boron nitride nanosheets for aerobic oxidative desulfurization. Chem Commun. 2016a;52(1):144-7. https://doi.org/10.1039/ c5cc07830j.

Wu P, Zhu W, Dai B, Chao Y, Li C, Li H, et al. Copper nanoparticles advance electron mobility of graphene-like boron nitride for enhanced aerobic oxidative desulfurization. Chem Eng J. 2016b;301:123-31. https://doi.org/10.1016/j.cej.2016.04.103.

Wu P, Dai B, Chao Y, Wu Y, Jiang W, Li C, et al. Graphene-like boron nitride anchored Brønsted acid ionic liquids as metal-free catalyst for advanced oxidation process. Mol Catal. 2017a;436:53-9. https://doi.org/10.1016/j.mcat.2017.04.008. 
Wu P, Yang S, Zhu W, Li H, Chao Y, Zhu H, et al. Tailoring $\mathrm{N}$-terminated defective edges of porous boron nitride for enhanced aerobic catalysis. Small. 2017b. https://doi.org/10. 1002/smll.201701857.

Wu Y, Wu P, Chao Y, He J, Li H, Lu L, et al. Gas-exfoliated porous monolayer boron nitride for enhanced aerobic oxidative desulfurization performance. Nanotechnology. 2018. https://doi.org/ 10.1088/1361-6528/aa9bc7.

Xiao J, Wang X, Fujii M, Yang Q, Song C. A novel approach for ultra-deep adsorptive desulfurization of diesel fuel over $\mathrm{TiO}_{2}-$ $\mathrm{CeO}_{2} / \mathrm{MCM}-48$ under ambient conditions. AIChE J. 2013;59(5):1441-5. https://doi.org/10.1002/aic.14085.

Xiao J, Wu L, Wu Y, Liu B, Dai L, Li Z, et al. Effect of gasoline composition on oxidative desulfurization using a phosphotungstic acid/activated carbon catalyst with hydrogen peroxide. Appl Energy. 2014;113:78-85. https://doi.org/10.1016/j.ape nergy.2013.06.047.

Xiao J, Sitamraju S, Chen Y, Watanabe S, Fujii M, Janik M, et al. Air-promoted adsorptive desulfurization of diesel fuel over TiCe mixed metal oxides. AIChE J. 2015;61(2):631-9. https://doi. org/10.1002/aic.14647.

Xiao X, Zhong H, Zheng C, Lu M, Zuo X, Nan J. Deep oxidative desulfurization of dibenzothiophene using a flower-like $\mathrm{WO}_{3}$ $\mathrm{H}_{2} \mathrm{O}$ catalyst in an organic biphasic system. Chem Eng J. 2016;304:908-16. https://doi.org/10.1016/j.cej.2016.07.022.

Xiong J, Yang L, Chao Y, Pang J, Wu P, Zhang M, et al. A large number of low coordinated atoms in boron nitride for outstanding adsorptive desulfurization performance. Green Chem. 2016;18(10):3040-7. https://doi.org/10.1039/c5gc02999f.

Xu J, Zhao S, Chen W, Wang M, Song Y-F. Highly efficient extraction and oxidative desulfurization system using $\mathrm{Na}_{7} \mathrm{H}_{2-}$ $\mathrm{LaW}_{10} \mathrm{O}_{36} \cdot 32 \mathrm{H}_{2} \mathrm{O}$ in bmim $\mathrm{BF} 4$ at room temperature. Chem-Eur J. 2012;18(15):4775-81. https://doi.org/10.1002/chem. 201102754.

Xun S, Zhu W, Chang Y, Li H, Zhang M, Jiang W, et al. Synthesis of supported $\mathrm{SiW}_{12} \mathrm{O}_{40}$-based ionic liquid catalyst induced solventfree oxidative deep-desulfurization of fuels. Chem Eng J. 2016;288:608-17. https://doi.org/10.1016/j.cej.2015.12.005.

Yang H, Jiang B, Sun Y, Zhang L, Huang Z, Sun Z, et al. Heterogeneous oxidative desulfurization of diesel fuel catalyzed by mesoporous polyoxometallate-based polymeric hybrid. J Hazard Mater. 2017;333:63-72. https://doi.org/10.1016/j.jhaz mat.2017.03.017.

Zhang M, Zhu W, Xun S, Li H, Gu Q, Zhao Z, et al. Deep oxidative desulfurization of dibenzothiophene with POM-based hybrid materials in ionic liquids. Chem Eng J. 2013;220:328-36. https:// doi.org/10.1016/j.cej.2012.11.138.
Zhang J, Wang A, Wang Y, Wang H, Gui J. Heterogeneous oxidative desulfurization of diesel oil by hydrogen peroxide: catalysis of an amphipathic hybrid material supported on $\mathrm{SiO}_{2}$. Chem Eng J. 2014a;245:65-70. https://doi.org/10.1016/j.cej.2014.01.103.

Zhang S, Zhang X, Jiang G, Zhu H, Guo S, Su D, et al. Tuning nanoparticle structure and surface strain for catalysis optimization. J Am Chem Soc. 2014b;136(21):7734-9. https://doi.org/10. 1021/ja5030172.

Zhang M, Hu T, Ren G, Zhu Z, Yang Y. Research on the effect of surfactants on the biodesulfurization of coal. Energy Fuel. 2017a;31(8):8116-9. https://doi.org/10.1021/acs.energyfuels. $7 \mathrm{~b} 01116$.

Zhang Y, Gu Y, Dong X, Wu P, Li Y, Hu H, et al. Deep oxidative desulfurization of refractory sulfur compounds with cesium salts of mono-substituted phosphomolybdate as efficient catalyst. Catal Lett. 2017b;147(7):1811-9. https://doi.org/10.1007/ s10562-017-2078-5.

Zhao H, Baker GA, Wagle DV, Ravula S, Zhang Q. Tuning taskspecific ionic liquids for the extractive desulfurization of liquid fuel. ACS Sustain Chem Eng. 2016;4(9):4771-80. https://doi. org/10.1021/acssuschemeng.6b00972.

Zhao R, Wang J, Zhang D, Sun Y, Han B, Tang N, et al. Deep catalytic oxidative desulfurization of model fuel based on modified iron porphyrins in ionic liquids: anionic ligand effect. ACS Sustain Chem Eng. 2017;5(3):2050-5. https://doi.org/10. 1021/acssuschemeng.6b02916.

Zhu H, Zhang S, Guo S, Su D, Sun S. Synthetic control of FePtM Nanorods $(\mathrm{M}=\mathrm{Cu}, \mathrm{Ni})$ to enhance the oxygen reduction reaction. J Am Chem Soc. 2013a;135(19):7130-3. https://doi.org/10.1021/ ja403041g.

Zhu $\mathrm{H}$, Zhang S, Huang Y-X, Wu L, Sun S. Monodisperse $\mathrm{MxFe}_{3-x} \mathrm{O} 4(\mathrm{M}=\mathrm{Fe}, \mathrm{Cu} \mathrm{Co}, \mathrm{Mn})$ nanoparticles and their electrocatalysis for oxygen reduction reaction. Nano Lett. 2013b;13(6):2947-51. https://doi.org/10.1021/n1401325u.

Zhu W, Wu P, Yang L, Chang Y, Chao Y, Li H, et al. Pyridiniumbased temperature-responsive magnetic ionic liquid for oxidative desulfurization of fuels. Chem Eng J. 2013c;229:250-6. https:// doi.org/10.1016/j.cej.2013.05.115.

Zhu W, Zhu G, Li H, Chao Y, Zhang M, Du D, et al. Catalytic kinetics of oxidative desulfurization with surfactant-type polyoxometalate-based ionic liquids. Fuel Process Technol. 2013d;106:70-6. https://doi.org/10.1016/j.fuproc.2012.07.003.

Zhu W, Dai B, Wu P, Chao Y, Xiong J, Xun S, et al. Grapheneanalogue hexagonal $\mathrm{BN}$ supported with tungsten-based ionic liquid for oxidative desulfurization of fuels. ACS Sustainable Chem Eng. 2015;3(1):186-94. https://doi.org/10.1021/ sc5006928. 